Abstracts of PhD Theses at Irish Universities 2006

\author{
Statistical Analysis of Gene Expression Profiles Obtained \\ by Microarray Experiments \\ EMMA HOLIAN \\ emma.holian@nuigalway.ie
}

This is an abstract of the $\mathrm{PhD}$ thesis Statistical Analysis of Gene Expression Profiles Obtained by Microarray Experiments written by Emma Holian under the supervision of Prof. John Hinde at the Department of Mathematics, National University of Ireland, Galway and submitted in June 2006.

Microarray technology measures genetic expression in the cells of a tissue sample and is often implemented to identify the functions of genes in an organism. The analysis of data produced from biological experiments such as microarrays leads to unique computational and statistical problems. The aim of this research is to first outline the statistical challenges that are faced at each stage of microarray data analysis, to briefly capture and summarise the vast spectrum of current methodology and research that has been initiated in order to embrace these problems, and to develop and implement new and unique techniques to address some issues that have not yet been dealt with.

In particular gene expression profiles obtained from time-course microarray experiments exhibit a unique opportunity to model the trends and correlations between longitudinal genetic expressions. The proposed Mixture-Regression Cluster Model is developed to model and cluster the genes into groups according to their expressions measured over time. This model is similar to that of the multivariate normal mixture model in that clusters are identified by the EM algorithm but is adapted to incorporate the flexibility of regression curves to fit the trends. In this way, additional features such as 
covariates, random effects and correlation structures can be incorporated into the model while potentially offering a considerable saving on the number of parameters required to model the trends.

The results from such modelling could provide very useful information on the molecular processes tested in these experiments. While the statistical modelling techniques developed here are widely applicable to model and cluster data obtained from any longitudinal study.

\title{
The Dynamics of Packets of Surface Gravity Waves in Smooth Inhomogeneous Media
}

\author{
Colm P. Howlin \\ c.p.howlin@lboro.ac.uk
}

This is an abstract of the PhD thesis The Dynamics of Packets of Surface Gravity Waves in Smooth Inhomogeneous Media written by Colm P. Howlin under the supervision of Prof. Eugene Benilov at the Department of Mathematics and Statistics in the University of Limerick and submitted in May 2006.

The dynamics of packets of surface gravity waves in smooth inhomogeneous media are governed by a nonlinear Schrödinger equation with variable coefficients. In the limits considered here the inhomogeneity is caused by the presence of slowly varying topography. There are two spatial scales in the problem: the horizontal scale of the inhomogeneity, or topography, and the distance over which nonlinearity and dispersion affect the packet. Accordingly, there are two limits where the problem can be analysed asymptotically: when the former scale is much larger than the latter and vice versa.

In examining the limit where the scale of nonlinearity or dispersion scale is much smaller than the topographic one it is found that the amplitude of a shoaling packet decays while its width grows. Furthermore, for waves of a given frequency, it is shown there exists a critical depth, $h_{c r}$, where if the packet reaches $h_{c r}$, it disperses.

It is found that for the limit where the scale of nonlinearity or dispersion scale is much larger than the topographic one, the packet undergoes rapid oscillations and also evolves slowly due to nonlinearity and dispersion. It is also demonstrated that the latter evolution 
is governed by a nonlinear Schrödinger equation with constant coefficients.

\section{REFERENCES}

[1] E. S. Benilov, J. D. Flanagan and C. P. Howlin, Evolution of packets of surface gravity waves over smooth topography, J. Fluid Mech. 533 (2005): 171-181.

[2] E. S. Benilov and C. P. Howlin, Evolution of packets of surface gravity waves over strong smooth topography, Stud. Appl. Math. 116 (2006): 289-301.

\section{An Investigation of Permanence and Exclusion in a Two-dimensional Discrete Time Competing Species Model PATRICK J. JOHNSON \\ patrick.johnson@ul.ie}

This is an abstract of the $\mathrm{PhD}$ thesis An Investigation of Permanence and Exclusion in a Two-dimensional Discrete Time Competing Species Model written by Patrick J. Johnson under the supervision of Dr. Mark E. Burke at the Department of Mathematics and Statistics, University of Limerick and submitted in October 2006.

In this work, a discrete version of the Lotka-Volterra equations [4] was used to model competition between two species. Denoting the populations of the two species at time $n$ by $x_{n}$ and $y_{n}$, respectively, it is possible to derive a discrete time two-dimensional quadratic system of equations represented by the map $T: R_{+}^{2} \rightarrow R_{+}^{2}$, where $T\left(x_{n}, y_{n}\right)=\left(x_{n+1}, y_{n+1}\right)$ with

$$
\begin{aligned}
& x_{n+1}=a x_{n}\left(1-x_{n}-s y_{n}\right), \\
& y_{n+1}=b y_{n}\left(1-y_{n}-t x_{n}\right),
\end{aligned}
$$

where $a$ and $b$ represent the growth rates of the species and $s$ and $t$ are the interspecific competition parameters. When $s$ and $t$ are both less than 1, the self-limiting terms of the map dominate the eventual evolution of the system. In contrast to this, when $s$ and $t$ are both greater than 1, competition between the species plays a more important role and thus has a more significant effect on the overall evolution of the system. The mixed case of one competition parameter greater than 1 and the other less than 1 is also possible. Depending on which situation is being examined it is possible for 
either the coexistence of both species or the extinction of one or other of the competing species to occur.

It is obvious that several outcomes are possible in this type of system but in general these outcomes can be classified under the group headings of "permanence" [2] and "competitive exclusion" [1]. Permanence occurs within a two species system when it is possible for both the species to stably coexist. Competitive exclusion on the other hand occurs within a competing species system when one of the species is driven to extinction.

A significant problem arising in this system is that not all initial trajectories starting in $R_{+}^{2}$ are feasible trajectories of the system. An initial point in $R_{+}^{2}$ is said to have a feasible trajectory if the initial point and all future iterates of the point, under the map $T$, remain nonnegative in both $x$ and $y$. Knowing whether or not there exists a feasible region and being able to calculate the structure of this region are vital prerequisites to any discussion of permanence and exclusion in the system.

The work of this thesis is concerned with investigating when feasible trajectories exist and under what conditions the phenomena of permanence and competitive exclusion are exhibited in the above discrete time system. The analysis of the system is carried out using the Method of Critical Curves as well as traditional methods in the fields of nonlinear dynamics and population dynamics. Since its introduction, the Method of Critical Curves has proven to be an important tool in the analysis of the global dynamical properties of non-invertible maps [3].

\section{REFERENCES}

[1] R. Armstrong, R. McGehee, Competitive exclusion, The American Naturalist 115(2) (1980): 151-170.

[2] J. Hofbauer, V. Hutson, W. Jansen, Coexistance for systems governed by difference equations of Lotka-Volterra type, J. Math. Biology 25 (1987): 553570 .

[3] C. Mira, L. Gardini, A. Barugola, J. C. Cathala, Chaotic dynamics in twodimensional noninvertible maps, World Scientific, Singapore, 1996.

[4] V. Volterra, Variations and fluctuations of the number of individuals in animal species living together, Journal du Conseil International pour l'Exploration de la Mer 3 (1928): 3-51. 


\title{
The Structure of Spectral Isometries on $C^{*}$-Algebras
}

\author{
Conal Ruddy \\ conal.ruddy@boiss.boi.ie
}

This is an abstract of the PhD thesis The Structure of Spectral Isometries on $C^{*}$-Algebras written by Conal Ruddy under the supervision of Dr. Martin Mathieu in the Department of Pure Mathematics, Queen's University Belfast, Northern Ireland and submitted in September 2006.

A famous question which Kaplansky asked is: If a linear mapping between Banach algebras is unital and maps invertible elements onto invertible elements, is it true that it is a Jordan homomorphism? A unital linear map $T: A \rightarrow B$ between unital Banach algebras $A$ and $B$ which preserves invertible elements has the property that $\sigma(T x) \subseteq \sigma(x)$ for all $x \in A$, where $\sigma(x)$ denotes the spectrum of an element $x$. Kaplansky's question is therefore connected with the study of linear mappings preserving the spectrum.

Definition A A linear mapping $T: A \rightarrow B$ between unital $C^{*}$ algebras $A$ and $B$ such that $T\left(x^{2}\right)=(T x)^{2}$ for all $x$ in $A$ is a Jordan homomorphism.

The case of a commutative, unital semi-simple Banach algebra is covered by the Gleason-Kahane-Żelazko Theorem stating that, if $A$ and $B$ are unital Banach algebras with $B$ commutative, semi-simple and $T: A \rightarrow B$ is unital, linear and sends invertible elements in $A$ to invertible elements in $B$, then $T$ is a homomorphism. Kaplansky's question was already known to be positive for $M_{n}$, the $n \times n$ matrices over $\mathbb{C}$, by work of Marcus and Moyls.

A more general question is when the linear mapping preserves not the entire spectrum, but just the spectral radius $r(x)$ of each element $x$.

Definition B A linear mapping $T: A \rightarrow B$ between unital $C^{*}$ algebras $A$ and $B$ with the property that $r(T x)=r(x)$ for all $x$ in $A$ is called a spectral isometry.

In our thesis we are concerned with the following conjecture.

Conjecture [Mathieu] Every unital surjective spectral isometry between unital $C^{*}$-algebras is a Jordan isomorphism. 
This would give a positive answer to Kaplansky's question, in the case of $C^{*}$-algebras, but would indeed be a stronger result in that case.

Definition C A linear mapping $T: A \rightarrow B$ between unital $C^{*}$ algebras $A$ and $B$ which satisfies the identity

$$
T(z x)=(T z)(T x) \text { for all } z \in Z(A) \text { and } x \in A
$$

is called $Z$-multiplicative.

A Jordan isomorphism between unital $C^{*}$-algebras is $Z$-multiplicative. However, this property is not known in general for a spectral isometry between unital $C^{*}$-algebras. $Z$-multiplicativity of a spectral isometry implies invariance of Glimm ideals. It is this fact which is the key in the proofs, so we consider spectral isometries which are $Z$-multiplicative.

Our main result is the following.

Theorem Every $Z$-multiplicative unital surjective spectral isometry from a unital $C^{*}$-algebra onto a unital separable $C^{*}$-algebra with Hausdorff spectrum is a Jordan isomorphism.

This covers, e.g., the case of $M_{n}(C(X))$, the $n \times n$ matrices with entries which are continuous functions on a compact Hausdorff space $X$ (separability is not needed in this case).

Our approach allows us to prove similar results for $C(X, D)$, where $D$ can be any of several $C^{*}$-algebras for which the conjecture holds.

We have an analogous result, replacing a separable $C^{*}$-algebra with Hausdorff spectrum by a continuous-trace $C^{*}$-algebra. Continuous-trace $C^{*}$-algebras have Hausdorff spectrum and are liminal. This allows us to drop the separability condition. However, we believe the $Z$-multiplicative assumption can be dropped and still have faith in Mathieu's Conjecture.

\section{REFERENCES}

[1] M. Mathieu and C. Ruddy, Spectral isometries, II, Contemp. Math., to appear. 


\section{Annihilating Polynomials and Stirling Numbers of the Second Kind \\ Stefan A. G. De Wannemacker \\ stefan.dewannemacker@gmail.com}

This is an abstract of the $\mathrm{PhD}$ thesis Annihilating polynomials and Stirling numbers of the second kind written by Stefan A. G. De Wannemacker under the supervision of David W. Lewis and Robert Osburn at the School of Mathematical Sciences, University College Dublin, Ireland and submitted in May 2006.

"Since it happens very often that series converge to the true value so slowly that they are no more use for the intended purpose than if they were in fact divergent, I have presented certain theorems in the first part of this treatise, by means of which one may arrive promptly at the values of those series which approximate most slowly of all: and indeed with that intention that Problems which depend upon quadratures can be considered for solution with the same authority as those which are reduced to affected questions. For here I do not examine which series are summable (as some may perhaps infer from the title) but by what methods we may come up with the values of those which are not able to be summed. ... [3]"

James Stirling (1730)

In his major work 'Methodus Differentialis: sive Tractatus de Summatione et Interpolatione Serierum Infinitarum' [2] James Stirling (1692-1770) studies the convergence of series and in particular transformations of these series with the aim of speeding up convergence. This often involved conversion of factorials into powers and vice versa and he drew up tables for this purpose. The numbers in these tables are now known as Stirling numbers. There are two different types of Stirling numbers, namely the Stirling numbers of the first kind and the Stirling numbers of the second kind.

In this thesis we focus on properties of Stirling numbers of the second kind and their applications to several areas of mathematics. Stirling numbers of the second kind already appeared in Algebraic Topology and Algebraic K-Theory. The purpose of this thesis is to further investigate properties of the Stirling numbers of the second kind and apply them to other areas of mathematics. 
The thesis is organized as follows. In a first chapter we provide the necessary tools for the remainder of the thesis. In the next chapter we prove that for any $k=1, \ldots, 2^{n}$ the 2 -adic order of the Stirling number $S\left(2^{n}, k\right)$ of the second kind is exactly $s_{2}(k)-1$, where $s_{2}(k)$ denotes the number of 1's among the binary digits of $k$. This confirms a conjecture of Lengyel [5]. In the next chapter we will prove a combinatorial conjecture of Wilf for an infinite number of values and discuss applications of this result to graph theory, multiplicative partition functions, and the irrationality of $p$-adic series [1].

Another surprising application of Stirling numbers of the second kind is to the algebraic theory of quadratic forms. In the last chapter we present a set of generators of the full annihilator ideal for the Witt ring of an arbitrary field of characteristic unequal to two satisfying a non-vanishing condition on the powers of the fundamental ideal in the torsion part of the Witt ring. This settles a conjecture of Ongenae and Van Geel. This result could only be proved by first obtaining a new lower bound on the 2-adic valuation of Stirling numbers of the second kind [4].

\section{REFERENCES}

[1] T. J. Laffey, R. Osburn, S. De Wannemacker, On a conjecture of Wilf, preprint 2006

[2] J. Stirling, Methodus Differentialis: sive Tractatus de Summatione et Interpolatione Serierum Infinitarum, London, 1730.

[3] I. Tweddle, James Stirling's Methodus Differentialis, Springer-Verlag, Berlin-Heidelberg-New York-Tokyo, 2003.

[4] S. De Wannemacker, Polynomials annihilating the Witt ring and 2-adic valuation of Stirling numbers, to appear in Math. Nachr. 2006.

[5] S. De Wannemacker, On the 2-adic orders of Stirling numbers of the second kind, INTEGERS 5(1), A:21 (2005). 